\title{
Os sinsignos icônicos nas 66 classes de signos: uma análise semiótica da fonte tipográfica Univers Revolved
}

Priscila Monteiro Borges

Resumo: O potencial analítico dos modelos de três e dez classes de signos de C. S. Peirce é reconhecido pelos pesquisadores da área. O mesmo não se pode dizer do sistema de 66 classes. Pouco conhecido, pois foi deixado em aberto por Peirce, e ainda é alvo de muitas discussões a respeito de sua aplicabilidade. Com o propósito de mostrar o seu potencial analítico, este artigo apresentará uma análise dos sinsignos icônicos extraída de uma análise completa de uma fonte tipográfica fundamentada no sistema de 66 classes de signos. Além de corresponder à parte empírica de uma pesquisa teórica, que propõe de um modelo analítico com as 66 classes, a análise mostra detalhes da relação entre sinsignos icônicos e qualissignos que não podem ser percebidos nos sistemas com menos classes, o que é uma evidência da contribuição do sistema de 66 classes a semiótica.

Palavras-chave: semiótica aplicada; C. S. Peirce; tipografia

Abstract: Iconic Sinsigns within Peirce's system of 66 classes of signs: a semiotic analysis of the typeface Univers Revolved - Although Peircean scholars have acknowledged the analytical potential of C. S. Peirce's models of three and ten classes of signs, such is not the case of his system of sixty-six classes. This system is practically unknown because Peirce did not conclude it, and its applicability to semiotic analysis has been discussed extensively. This paper testifies to the analytical potential of the system of sixty-six classes by presenting an analysis of the Iconic Sinsigns extracted from a full analysis of a typeface. The paper presents the empirical part of a theoretical study that proposes an analytical model of Peirce's sixty-six classes of signs. In particular, it shows the relationship between Iconic Sinsigns and Qualisigns at a level of detail that is indiscernible in his other systems with fewer classes, providing evidence of the relevance of Peirce's system of sixty-six classes of signs for a better understanding of semiotics.

Keywords: applied semiotics; C. S. Peirce; typography. 


\section{Introdução}

Este trabalho é parte de uma pesquisa que busca desenvolver um método de análise semiótica utilizando o sistema de 66 classes de signos de C. S. Peirce, a sua aplicação em análises tem o papel de testar empiricamente o modelo concebido teoricamente. Apresentaremos, neste texto, parte de uma análise feita com a fonte tipográfica Univers Revolved que se refere aos sinsignos icônicos. A análise dos sinsignos é fundamentada na análise dos qualissignos, que a antecede. Por isso, resgataremos os conceitos desenvolvidos na análise dos qualissignos, que são indispensáveis para a análise dos sinsignos icônicos. Os interessados em conhecer a análise completa podem encontra-la na tese de doutorado de Borges (2010).

A semiótica peirceana tem como proposta desenvolver um modelo capaz de explicar toda a multiplicidade de fenômenos no mundo. Ela foi criada como uma disciplina filosófica constituída de um padrão de classificação que indica logicamente todas as semioses possíveis e tem sido amplamente aplicada aos mais variados signos.

Peirce concebeu a semiótica a partir de um processo sistemático de observação e experiência com os fenômenos. Desse processo, ele concluiu que existiam três categorias fundamentais presentes em todos os fenômenos: primeiridade, secundidade e terceiridade. Ideias de primeiridade estão relacionadas à originalidade, acaso, potência, qualidade, mônada, indeterminação. É aquilo que é em si mesmo, sem referência a nada. A secundidade está aliada à experiência de esforço, oposição, dualidade, conflito, resistência, ação bruta. Ela estabelece uma relação com um segundo termo, mas sem referência a um terceiro. É uma experiência do instante, portanto, única e particular. A terceira categoria consiste na ideia de lei, pensamento, hábito, temporalidade. Ela é a mediação, elemento capaz de estabelecer a ligação entre outros dois termos. Envolve, portanto, generalidade, propósito, continuidade e representação (SANTAELLA, 2000, p. 8).

As categorias fenomenológicas são encadeadas de modo que a primeira é a única independente, a segunda depende da primeira e a terceira depende da segunda e da primeira. Primeiridade, secundidade e terceiridade também foram chamadas de qualidade, relação e representação. Peirce explica a relação de dependência entre elas dizendo que uma relação só é possível quando existem qualidades e uma representação depende tanto de qualidades quanto de relações (EP 2, p. 270 [1903]).

O signo é um elemento da terceira categoria, pois ele é constituído por uma relação triádica e tem a função de representar. Os três termos que compõem a relação no signo são: o fundamento, o objeto e o interpretante do signo. Segundo Peirce,

um signo intenta representar, em parte, pelo menos, um objeto que é, portanto, num certo sentido, a causa ou determinante do signo, mesmo que o signo represente o objeto falsamente. Mas dizer que ele representa seu objeto implica 
que ele afete uma mente de tal modo que, de certa maneira, determina, naquela mente, algo que é mediatamente devido ao objeto. Essa determinação da qual a causa imediata ou determinante é o signo e da qual a causa mediada é o objeto pode ser chamada de interpretante. (CP 6.347 [1909] apud SANTAELLA, 2001, p.42-43)

A primeira classificação dos signos de Peirce mostra apenas um aspecto sob o qual o signo pode ser analisado: sua referencialidade, ou seja, a relação entre signo e objeto. Quando a relação é de primeiridade temos um ícone, de secundidade, um índice, e de terceiridade, um símbolo. Apesar de amplamente divulgados e aplicados, esses três conceitos representam apenas o início da semiótica de Peirce.

As dez classes de signos foram criadas a partir da análise de três aspectos do signo: o signo em si, a relação entre signo e objeto e a relação entre signo e interpretante. Cada aspecto é denominado uma tricotomia que apresenta três modalidades, conforme as categorias fenomenológicas. O signo em si pode ser um qualissigno, sinsigno ou legissigno. A relação entre signo e objeto pode ser icônica, indicial ou simbólica. Por fim, a relação entre signo e interpretante pode ser remática, dicente ou argumental.

Já as 66 classes surgem da divisão dos objetos em dinâmico e imediato e dos interpretantes em imediato, dinâmico e final, elevando para dez os aspectos a serem analisados no signo: objeto dinâmico [OD], objeto imediato [OI], signo em si [S], relação do signo com o objeto dinâmico [S-OD], interpretante imediato [II], interpretante dinâmico [ID], relação do signo com o interpretante dinâmico [S-ID], interpretante final [IF], relação do signo com o interpretante final [S-IF] e, finalmente, relação entre objeto dinâmico, signo e interpretante final [OD-S-IF]. Como no sistema de dez classes, cada tricotomia pode ter três modalidades, gerando um sistema com 66 classes de signos. Essa classificação foi sugerida por Peirce, mas não pode ser completamente desenvolvida por ele. Consequentemente, é um assunto pouco explorado e ainda cheio de controvérsias. (Sobre as 66 classes de signos, ver: WEISS e BURKS, 1945; FARIAS e QUEIROZ, 2003, 2004, 2006; SANDERS, 1970; MERKLE, 2001; ROMANINI, 2006; BORGES, 2010).

\section{Sinsignos icônicos no sistema de 10 e de 66 classes de signos}

Quando o signo em si é de secundidade e a relação entre signo e objeto dinâmico é de primeiridade temos o sinsigno icônico. Por ser um sinsigno, suas qualidades estão materializadas em algum meio, sendo passíveis de experiência sensível. Como é icônico, seu poder representativo é dado por similaridade, suas qualidades existentes podem assemelhar-se a outras e, assim, ele é capaz de referir-se a algo. Apesar de esse signo compartilhar qualidades com o objeto, é sua singularidade que o torna capaz de representar. É a forma como suas características são apresentadas naquele instante que o torna similar ao objeto (EP 2, p. 294 [1903]). 
O sistema de dez classes de signos descreve apenas uma classe de sinsigno icônico. Ela é a segunda classe do sistema, precedida apenas pela classe do qualissigno. Peirce a descreve como:

qualquer objeto de experiência na medida em que alguma de suas qualidades faz com que ele determine a ideia de um Objeto. Sendo ele um Ícone, e desse modo um signo por pura semelhança de qualquer coisa com que ele possa se assemelhar, só pode ser interpretado como signo de essência, ou Rema. Ele incorporará um Qualissigno. (EP 2, p. 294; CP 2, p. 255 [1903])

O sistema de 66 classes de signos, por sua vez, descreve três classes de sinsignos icônicos. As três classes diferenciam-se por terem objeto dinâmico e objeto imediato de naturezas diferentes. As tricotomias dos objetos precedem a tricotomia do signo em si, estabelecendo uma ordem de determinação que vai do objeto dinâmico [OD], para o objeto imediato [OI] e, em seguida, para o signo em si [S]. O objeto dinâmico independe do signo, que funciona como agente determinador do signo (EP 1, p. xxxix). Ele pode ser abstrativo, concretivo ou coletivo. Já o objeto imediato faz parte do signo, ele corresponde a uma dica dada pelo signo sobre o seu objeto dinâmico. Ele pode ser descritivo, denominativo ou distributivo.

A natureza dos objetos mostra uma convencionalidade crescente nos sinsignos icônicos. A primeira classe de sinsigno icônico encontrada apresenta ambos os objetos de secundidade, isto é, objeto dinâmico concretivo e objeto imediato designativo. Sinsignos concretivos designativos descrevem fatos atuais e singulares fruto de uma relação existencial entre objeto e signo. A segunda classe apresenta objeto dinâmico de terceiridade ou coletivo e objeto imediato de secundidade, designativo. Sinsignos coletivos designativos dizem respeito a fatos que indicam a existência de uma lei atuando no objeto. Por fim, a terceira classe apresenta os dois objetos de terceiridade, objeto dinâmico coletivo e objeto imediato distributivo. Sinsignos coletivos distributivos descrevem à própria lei do objeto que afeta o signo.

As diferenças entre esses sinsignos icônicos estão relacionadas à gradativa convencionalidade, observada nos seis qualissignos do sistema de 66 classes (BORGES, 2010 e 2012). Eles descrevem qualidades possíveis do signo de acordo com a natureza dos seus objetos dinâmico e imediato. Portanto, a análise de cada um dos sinsignos icônicos incorporará os qualissignos que possuem os objetos correspondentes. Veremos a seguir como isso ocorre.

\section{Sinsignos icônicos da fonte tipográfica Univers Revolved}

A fonte tipográfica Univers Revolved foi concebida por Ji Lee, um designer coreano radicado nos Estados Unidos. Seus caracteres foram construídos a partir da rotação em $360^{\circ}$ das letras maiúsculas da fonte Univers em torno de um eixo vertical, colocado 
no ponto mais a esquerda do caractere (ver figura 1,2). Desse modo, Lee constrói caracteres alfabéticos tridimensionais e simétricos que podem ser lidos em qualquer direção (ver figuras 3, 4, 5, 6). Esses caracteres possibilitam escrever textos tridimensionais e experimentar diferentes formas de leitura.

A fonte Univers Revolved foi escolhida para análise porque ela reinventa a relação entre as linguagens visual e verbal de tal modo que transforma o texto escrito em texto cifrado. Para compreender o significado dessa fonte e de seus textos é necessário decifrar sua imagem. Consequentemente, os sinsignos icônicos serão analisados no que diz respeito à capacidade representativa de suas formas visuais.

Para fazer a análise da fonte tipográfica Univers Revolved utilizamos o modelo de 66 classes combinado às matrizes da linguagem visual descritas por Santaella (2001, p. 185260). A relação do modelo com as matrizes fez-se necessária porque a semiótica peirceana é uma lógica do método investigativo, ela não fornece informação específica sobre o funcionamento da linguagem visual. Como no livro Matrizes da Linguagem, Santaella (2001) apresenta uma teoria da linguagem visual fundamentada na filosofia peirceana, ela foi escolhida como sendo a teoria mais adequada ao método adotado nesta pesquisa.

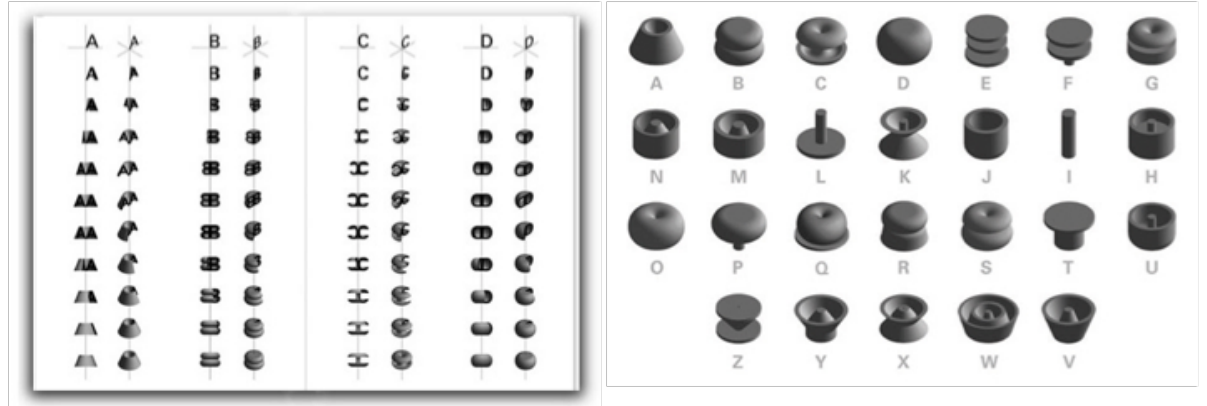

Fig. 1. Modo de construção dos caracteres da fonte Univers Revolved.

Fig. 2. Vista superior dos caracteres da fonte Univers Revolved.
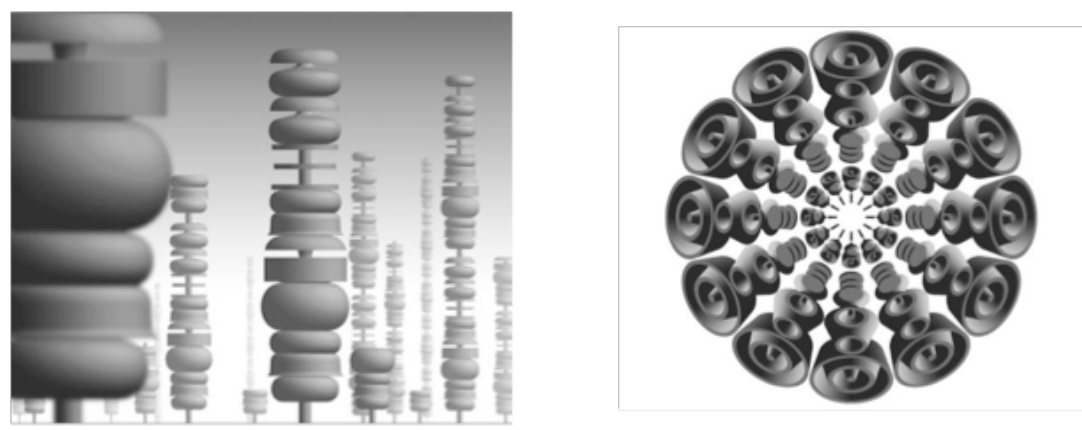

Fig. 3. Progress (Progresso). Texto escrito com a fonte Univers Revolved. Fig. 4. Wake up! (Acorde!) Texto escrito com a fonte Univers Revolved. 

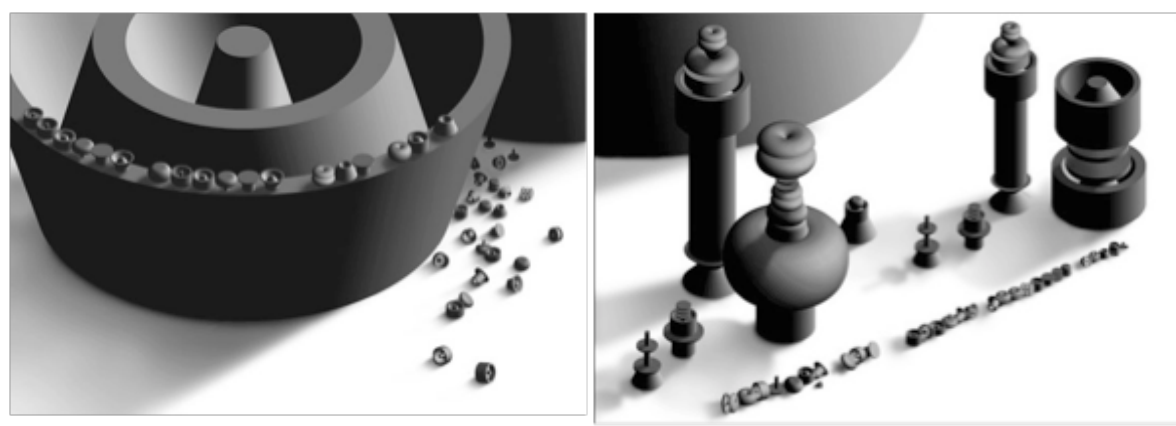

Fig. 5. "Humpty Dumpty em um muro se sentou, Humpty Dumpty lá de cima despencou." (Caroll, 1980, p.192). Texto escrito com a fonte Univers Revolved.

Fig. 6. "Erguê-lo não podem os cavalos do rei, nem mesmo todos os cavaleiros do rei, também." (Caroll, 1980, p.192) Texto escrito com a fonte Univers Revolved.

Segundo Santaella (2001), o poder de referencialidade da linguagem visual pode apresentar-se de três modos: forma não-representativa, forma figurativa e forma representativa. As formas não-representativas são autorreferentes e enfatizam a apresentação do signo em si mesmo. As figurativas, por sua vez, apresentam uma forma definida que se distingue do fundo e cuja organização interna e externa pode ser percebida. Finalmente, as formas representativas são capazes de representar coisas abstratas e gerais. Na análise que se segue observaremos cada classe de sinsigno icônico conforme esses três modos de referencialidade.

\section{Sinsigno icônico concretivo designativo}

Como essa classe de sinsignos icônicos é precedida por objetos pertencentes à categoria da secundidade ([OD]: concretivo; [OI] designativo), ela indica que o registro do objeto ocorre por uma conexão existencial. Como a relação entre signo e objeto é icônica, esse signo tem qualidades semelhantes às qualidades do objeto a que ele se refere.

Objetos dinâmicos concretivos podem ser encontrados em duas classes de qualissignos: no qualissigno concretivo descritivo e no qualissigno concretivo designativo. Para entendermos esse tipo de sinsigno icônico, precisamos retomar à análise feita nessas classes.

No qualissigno concretivo descritivo (BORGES, 2010, p. 169) vimos a possibilidade da fonte Univers Revolved apresentar qualidades visuais particulares, produzindo um universo próprio. Os caracteres dessa fonte apresentam poucas qualidades visuais semelhantes a dos caracteres da fonte Univers, da qual eles derivam. Consequentemente, a relação entre a fonte e o alfabeto não pode ser percebida por semelhança de qualidade, mas sim por uma relação existencial.

A fonte Univers Revolved é fruto de um comando digital que rotaciona uma forma plana em torno de um eixo, gerando uma forma com três dimensões. As fontesUnivers e a Univers Revolved estabelecem uma relação existencial, pois, sem uma não seria 
possível criar a outra. O comando digital modifica as qualidades visuais da fonte Univers, dificultando a relação por semelhança de qualidades, mas a forma dos caracteres tridimensionais é determinada pela forma dos caracteres modelo.

O qualissigno concretivo designativo (BORGES, 2010, p. 175) informa a possibilidade de certas qualidades visuais do signo indicar o seu modo de produção. Se um comando digital aplicado a uma forma plana gera vetores que descrevem uma imagem, então, qualidades como a precisão de traços e degrades e a capacidade da imagem adequar-se a qualquer escala sem perda de qualidade podem indicar uma imagem digital vetorial. A materialização dessas qualidades descritas matematicamente em formas visuais ocorre no nível do sinsigno icônico concretivo designativo. Isso corresponde à forma nãorepresentativa desse signo, no qual um gesto em ato produz uma qualidade que indica o acontecimento singular desse gesto (SANTAELLA, 2001, p. 219).

Com relação ao registro dessa imagem, esse primeiro sinsigno icônico descreve um registro por conexão dinâmica que mantém algum tipo de semelhança qualitativa entre signo e objeto, chamado de registro imitativo. Nesse tipo de registro a semelhança de qualidade não é a única responsável pela representatividade do signo, o signo deve existir no mesmo tempo ou espaço que o seu objeto. Observamos na fonte Univers Revolved que os caracteres apresentam uma tênue semelhança com os caracteres alfabéticos. Essa semelhança formal possibilita descobrir qual letra o objeto tridimensional representa. Se os caracteres da Univers Revolved não estiverem efetivamente ocupando o lugar de letras em textos ou em uma fonte digital, eles não serão entendidos como caracteres alfabéticos, pois há um baixo grau de similaridade visual entre eles. Por outro lado, os caracteres da fonte Univers Revolved podem facilmente se assemelhar a outros objetos tridimensionais.

No que se refere à forma representativa da linguagem visual, observamos a capacidade de representar por semelhança, que corresponde ao primeiro modo representativo das formas visuais. Esse modo representativo aparece no sinsigno icônico concretivo designativo como representação imitativa com a predominância da função mimética. Um signo pode representar por semelhança mesmo que ele seja um signo convencional. A fonte Univers Revolved pode exercer essa função quando a imagem de seus caracteres, isoladamente, ou compostos em imagens-textos, assemelha-se a algum objeto e passa a representá-lo. Essa representação antecede a identificação de caracteres alfabéticos e a relação da imagem com a linguagem verbal.

\section{Sinsigno icônico coletivo designativo}

Essa classe de sinsignos icônicos se diferencia da primeira por ter objeto dinâmico coletivo. Sabemos que coletivo se refere a algo da natureza de lei. Como três classes de qualissignos anteriores apresentaram objetos dinâmicos coletivos, devemos recorrer a eles. A primeira delas, qualissigno coletivo descritivo, diz respeito às leis naturais 
da qualidade, nesse caso, leis naturais do meio digital que permitem a criação de imagens digitais. A segunda classe, qualissigno coletivo designativo, diz respeito às leis do acaso, que descrevem casos singulares das leis naturais do meio digital. Por fim, as abstrações da lei, na classe de qualissigno coletivo distributivo, descrevem os modos de representar as leis da natureza como, por exemplo, a linguagem matemática e as leis da geometria.

Essas três classes de qualissignos apresentam leis que regem o funcionamento das formas visuais digitais (leis naturais da qualidade, lei do acaso e abstrações da lei). No sinsigno icônico coletivo designativo também encontramos algumas leis que explicam o funcionamento da linguagem visual, são as leis físicas e fisiológicas do gesto. No entanto, as leis, nessa classe, não são leis gerais das formas visuais digitais, mas dizem respeito às leis específicas a um determinado gesto. Como estamos analisando um signo criado digitalmente, não encontramos leis do gesto manual, mas sim leis dos gestos digitais. A rotação da forma plana em torno de um eixo é um gesto digital. O contorno e as qualidades visuais da imagem gerada são determinados por leis digitais que comandam o movimento de rotação. Portanto, as leis físicas e fisiológicas que regem o gesto produtor dessa fonte são leis digitais que determinam a forma de todos os caracteres da fonte, dando-lhes características de grupo.

O comando de rotacionar cria os caracteres da Univers Revolved, mas, para formar esses caracteres, não basta a ação ocasionada pelo comando digital, é necessário que haja o registro desse gesto para que os caracteres possam ser vistos e repetidos, caso contrário eles teriam a duração de um instante e desapareceriam. Os caracteres em um arquivo de fonte tipográfica digital são o registro desse gesto.

O tipo de registro que observamos nessa classe é o registro físico. As fotografias são um bom exemplo de imagens produzidas pelo registro físico. Apesar de haver uma conexão existencial entre a imagem fotografada e o seu objeto que caracteriza o registro, ele não é apenas fruto de um choque brutal de matérias. Várias regras regem o funcionamento da máquina fotográfica, permitindo-a registrar uma cena, elas estão relacionadas ao objeto dinâmico coletivo.

Observamos que o registro físico na fonte Univers Revolved é caracterizado pelo registro da transformação das formas planas em tridimensionais. Esse registro não preserva todas as qualidades visuais do seu referente, há um código que atua e confere outras qualidades ao signo interferindo no seu grau de semelhança. O mesmo acontece nas imagens de tomografias computadorizadas ou ressonâncias magnéticas, para as quais é necessário o conhecimento específico do sistema de codificação que registra a imagem para sua compreensão.

Também no caso analisado, é preciso conhecer a regra que foi aplicada aos caracteres para compreender a relação indicial entre a forma do caractere da fonte Univers e a imagem tridimensional resultante. Como o registro físico transforma as qualidades visuais da fonte original, ele não explicita a semelhança visual entre cada forma tridimensional e o caractere alfabético que a determina. No entanto, ele garante a relação existencial 
entre eles e assegura que as imagens tridimensionais foram determinadas por uma forma plana. Portanto, ele determina uma correspondência entre as imagens tridimensionais e os caracteres alfabéticos.

Mas qual é o potencial do sinsigno icônico coletivo designativo funcionar como uma forma visual representativa? Seu potencial representativo é imitativo, assim como o potencial da classe de sinsigno icônico concretivo designativo. A diferença da representação imitativa nessas duas classes é dada pelos diferentes objetos dinâmicos delas. Enquanto a primeira classe de sinsignos icônicos apresenta objeto dinâmico concretivo caracterizado pelo gesto em ato, ou seja, cada ação do comando digital é considerada individualmente. Nesta segunda classe, o objeto dinâmico é coletivo e caracterizado pela modalidade das leis físicas e fisiológicas do gesto. Isto é, a semelhança de qualidade entre os caracteres das fontes mostra que eles fazem parte de um grupo, formam um conjunto e essa informação é fundamental para a identificação delas com o alfabeto, que é formado por um conjunto de caracteres.

Se no sinsigno icônico anterior cada caractere podia representar qualquer objeto com forma visual semelhante, nesse sinsigno icônico o conjunto de caracteres pode formar uma imagem com forma visual semelhante a de algum outro objeto. Como os caracteres da fonte Univers Revolved passaram por um processo de criação que modifica as qualidades visuais dos caracteres alfabéticos de referência, o reconhecimento dessas formas tridimensionais como representativas do alfabeto romano depende do conhecimento do seu modo de criação. Mas o signo não deixa de ter poder representativo enquanto essas informações não são alcançadas, ele pode representar mimeticamente devido às semelhanças de formas visuais com outros objetos conhecidos.

\section{Sinsigno icônico coletivo distributivo}

A última classe de sinsignos icônicos é composta exclusivamente por objetos no nível de terceiridade ([OD]: coletivo; [OI]: distributivo). Eles são da natureza de uma lei e dependem de uma sequência lógica de raciocínio para serem compreendidos. Essa mesma combinação de objetos foi discutida na classe de qualissigno coletivo distributivo. Nela identificamos leis abstratas responsáveis pela criação de formas visuais, isto é, um sistema numérico que ao ser processado gera uma imagem. Enquanto no qualissigno descrevemos um sistema numérico capaz de criar formas visuais que não existem efetivamente, mas apenas como possibilidade, nos sinsignos temos um signo efetivamente materializado. No sinsigno icônico coletivo distributivo, observamos, portanto, as réplicas como instâncias da lei. Isto é, as formas visuais criadas sinteticamente (chamadas de réplicas) apontam à existência de leis convencionais que comandam a produção da imagem.

Com relação à forma figurativa, observamos nessa classe o registro como convenção. Apesar de a figura ser "efetivamente determinada pelo objeto que ela indica" (SANTAELLA, 
2001, p. 237), no registro como convenção há uma regra ou lei, isto é, normas de representação figurativa determinam como esse registro será realizado.

Como as figuras são produzidas a partir de regras convencionais, a similaridade apresentada entre signo e objeto pode não ser apenas de aparência visual como a que encontramos no registro imitativo. No registro como convenção, a imitação pode se dar por uma semelhança entre as relações internas do signo e as relações internas do objeto denotado. Para perceber essa semelhança não basta acuidade visual, é preciso conhecer e aprender tanto as regras convencionais que regem o registro como as leis que formam o próprio objeto representado. Sabemos que as leis que regem o registro de fontes tipográficas são leis dos sistemas de escrita que adquiriram características particulares no processo de criação da fonte.

O sistema singular de escrita da fonte Univers Revolved busca desautomatizar a leitura verbal e fazer ver a escrita sob perspectivas diferentes. Ela faz isso ao transformar os caracteres planos em tridimensionais e a linearidade do sistema de escrita em espacialidade. Desse modo, ela singulariza a convenção da linguagem escrita, criando um estilo próprio que foi descrito na classe de qualissigno coletivo designativo.

Já a forma tridimensional dos caracteres se assemelha a peças, partes de objetos volumétricos do dia a dia, podendo representar brinquedos, peças de móveis, prédios, etc. Nesse caso, a representação visual se dá porque os caracteres da fonte são reconhecidos como sendo objetos tridimensionais construídos digitalmente. A possibilidade de ser reconhecido como objeto tridimensional foi descrito pelo qualissigno coletivo distributivo.

Como essa fonte propõe a desautomatização da escrita e da leitura e o rompimento das regras da linguagem alfabética, observamos nela uma flexibilização no que diz respeito ao registro por convenção. Não há uma convenção que determine como os caracteres devem ser compostos. Essas regras podem variar priorizando ora a linguagem verbal, ora a linguagem visual, utilizando regras de apenas uma dessas linguagens, ou combinando ambas. A semelhança dos caracteres a objetos ou partes de objetos sugere que esses textos componham imagens figurativas que se assemelham a objetos conhecidos.

No que se refere à capacidade representativa do signo, observamos uma complexificação da representatividade das formas visuais. Enquanto as formas visuais no sinsigno icônico coletivo designativo representam por semelhança, no sinsigno icônico coletivo distributivo elas representam por cifra. Isto é, as formas visuais dessa classe "não guardam mais qualquer analogia com o objeto representado, de modo que essas figuras adquirem natureza hermética e fechada" (SANTAELLA, 2001, p. 253).

As cifras são figuras aparentemente singulares que se referem às ideias gerais e abstratas diferentes da ideia geral das figuras que elas indicam. Para serem entendidas, elas precisam ser decodificadas. A estrutura codificada dessas formas visuais está relacionada aos objetos da terceira categoria encontrados nessa classe. Mas como se trata de um sinsigno icônico, apesar de ter uma estrutura codificada, a capacidade representativa 
desse signo é dada por semelhança. Portanto, a cifra que encontramos no sinsigno icônico coletivo distributivo é a cifra por analogia. Nela as formas visuais mantêm uma analogia com o que elas representam, mas a semelhança não é explícita, pois as formas são enigmáticas. A analogia só é percebida quando as formas do signo e do objeto são confrontadas e a cifra é revelada.

No caso da fonte Univers Revolved a forma visual do texto mantém analogia com aquilo que o texto verbal significa. A forma enigmática está no fato do texto apresentar semelhança à forma dos objetos que eles representam. Contudo, essa analogia não é evidente, pois a imagem não é uma mimese do seu objeto. Quando o texto é decifrado, a analogia aparece claramente. Por exemplo, no texto Progress (Progresso, fig. 3), observamos uma série de torres que representam arranha-céus e o progresso da industrialização. Nos textos sobre Humpty Dumpty (fig. 5 e 6) também não é clara a analogia visual, mas com o auxílio da legenda os elementos visuais são identificados. O mesmo acontece com o texto Wake up! (Acorde!, fig. 4) que mostra uma imagem semelhante a uma caixa de som.

\section{Conclusão}

Apesar de serem signos existentes, o processo de significação do sinsigno icônico é incerto, baseado em hipóteses, capaz de sugerir semelhanças e produzir um significado dado por uma espécie de simpatia, atração. Seu significado é produzido no nível do sentimento, mostra apenas uma tendência que poderá se efetivar nos sinsignos indiciais e dicentes.

Supondo que a experiência com a fonte Univers Revolved pode levar a maior compreensão de seu funcionamento como um sistema de escrita, podemos perceber como as qualidades de cada um dos sinsignos icônicos possibilitam a compreensão de que as imagens tridimensionais representam o alfabeto romano.

A primeira classe de sinsigno icônico, descritivo designativo, apresenta qualidades relacionadas ao gesto criador da imagem, isto é, qualidades que podem indicar o movimento preciso de rotação de uma forma plana. As qualidades volumétricas das imagens sugerem que diferentes formas planas foram rotacionadas $360^{\circ} \mathrm{em}$ torno de um eixo. Poderá ser percebida a relação das formas planas com as letras e consequentemente a ideia de que as imagens mostram o alfabeto romano. A partir de então, a imagem tenderia a representar o alfabeto.

Por sua vez, o sinsigno icônico coletivo designativo apresenta qualidades que mostram as leis físicas e fisiológicas do gesto. A tridimensionalidade dos caracteres funciona como registro de um comando digital de rotação. Precisão dos traços, contornos, simetria e tonalidade dos caracteres assemelham-se às qualidades de imagens digitais, portanto, supõe-se que as imagens foram criadas digitalmente por um comando de rotação. Precisão e semelhança das qualidades de todos os caracteres sugerem um estilo visual tipográfico próprio. A fonte apresenta modo particular de traçar caracteres, formam uma escritura que tende a ser também tridimensional. 
Finalmente, no sinsigno icônico coletivo distributivo, temos qualidades que são leis. Nesse caso, não falamos de qualidades próprias de um acontecimento singular, mas qualidades de um sistema visual ordenado por leis. Portanto, as formas tridimensionais são caracteres de uma fonte tipográfica cujas letras e composição do texto possuem características particulares. Como as formas dos caracteres assemelham-se a partes de objetos tridimensionais, supõe-se que eles possam formar imagens gráficas. Textos diagramados multidirecionalmente podem compor imagens gráficas, sugerindo que juntos imagens e palavras expressam uma ideia. Então, o significado de um texto pode ser constituído de linguagem visual e verbal. Letras tridimensionais e diagramação multidirecional tendem a criar um sistema de escrita próprio.

$\mathrm{Na}$ descrição do sinsigno icônico no sistema de dez classes de signos, Peirce deixa claro que esses signos incorporam um qualissigno (EP 2, p. 294; CP 2, p. 255). Apesar de sua afirmação, a ideia de que o sinsigno icônico incorpora um qualissigno é vaga nesse sistema que apresenta apenas uma classe de qualissigno e uma classe de sinsigno icônico, pois tanto as qualidades possíveis quanto as qualidades experienciadas não são discriminadas. Consequentemente, qualquer qualidade experienciada poderia incorporar qualquer qualidade possível.

Quando analisamos os sinsignos icônicos no sistema de 66 classes, percebemos que uma qualidade existente não incorpora qualquer qualidade possível do signo, mas sim aquelas qualidades relacionadas à própria qualidade existente. A distinção de seis qualissignos e de três sinsignos icônicos leva à compreensão mais detalhada de quais qualissignos tornam possível a existência de uma determinada qualidade e de como ela poderá representar por semelhança.

O sistema de 66 classes contribui à descrição mais precisa das relações de determinação sígnica, mostrando a complexidade do processo representativo. Na tipografia, as qualidades visuais são fundamentais ao processo de significação, são elas que possibilitam o reconhecimento de um alfabeto e a associação da mancha tipográfica a outros elementos gráficos visuais, construindo significado verbal e visual.

Priscila Monteiro Borges é doutora em Comunicação e Semiótica pela PUC-SP e professora no curso de Comunicação Social da Universidade Federal de Ouro Preto - UFOP. É diretora executiva do Centro Internacional de Estudos Peirceanos da PUC-SP, pesquisadora do Centro de Convergência de Novas Mídias na UFMG e vicesecretária geral da International Association for Semiotics Studies - IASS-AIS.

primborges@gmail.com 


\section{Referências}

BORGES, Priscila M. Mensagens cifradas: a criação de linguagens diagramáticas. Tese de doutorado em Comunicação e Semiótica. São Paulo, Programa de Comunicação e Semiótica, Pontifícia Universidade Católica de São Paulo, 2010.

BORGES, Priscila M. "Os Qualissignos nas 66 classes de signos de C.S.Peirce: uma análise semiótica da fonte tipográfica Univers Revolved". In: ADAMI, Antonio; HOHLFELDT, Antonio Carlos (orgs.). IX LUSOCOM Lusofonia e Interculturalidade. 1. ed. São Paulo, Intercom, p. 604-627, 1 vol, 2012.

CARROLL, Lewis. As aventuras de Alice. 3ed. São Paulo, Summus, 1980.

FARIAS, Priscila; QUEIROZ, João (2003). On diagrams for Peirce's 10, 28, and sixty-six classes of signs. Semiotica, v. 147, 2003.

10cubes and 3N3: Using interactive diagrams to investigate Charles Peirce's classifications of signs. Semiotica, v. 151, 2004.

. Images, diagrams, and metaphors: Hypoicons in the context of Peirce's sixty-six-fold classification of signs. Semiotica, v. 162, 2006.

LEE, Ji. Univers Revolved: A Three-Dimensional Alphabet. New York, Harry N. Abrams, 2004.

MERKLE, Luiz Ernesto. Disciplinary and Semiotic Relations across Human-Computer Interaction. Tese de doutorado em Ciência da Computação. London, Graduate Program in Computer Science, University of Western Ontario, 2001.

PEIRCE, Charles Sanders. Collected Papers of Charles S. Peirce. Cambridge, Harvard Univ. Press, 1-8 vol, 1931/1958. (Citado como CP, seguido do volume e página)

. The Essential Peirce. Selected Philosophical Writings. Bloomington, Indiana University Press, 1-2 vol, 1992/1998. (Citado como EP, seguido do volume e página)

ROMANINI, Anderson Vinícius. Semiótica Minuta: Especulações sobre a gramática dos signos e da comunicação a partir da obra de C.S.Peirce. Tese de doutorado em Ciências da Comunicação. São Paulo, Escola de Comunicação e Artes, Universidade de São Paulo, 2006

SANDERS, Gary. Peirce's sixty-six signs? Transactions of the Charles S. Peirce Society, v. 6, n. 1, 1970.

SANTAELLA, Lucia. Teoria Geral dos Signos. Como as linguagens significam as coisas. 2. ed. São Paulo, Pioneira, 2000.

Matrizes da Linguagem e do Pensamento: sonora, visual, verbal. São Paulo, Iluminuras, 2001.

WEISS, Paul; Burks, Arthur. Peirce's sixty-six signs. Journal of Philosophy, v. 42, n. 14, jul. 5, 1945.

Artigo recebido em abril

e aprovado em setembro de 2013. 